\title{
Sensor technology: a critical cutting edge of exposure science
}

\author{
Richard E. Peltier $\mathbb{D}^{1} \cdot$ Timothy J. Buckley ${ }^{2}$
}

Received: 17 August 2020 / Revised: 11 September 2020 / Accepted: 11 September 2020 / Published online: 23 September 2020

(c) Springer Nature America, Inc. 2020

We begin with a useful thought experiment: what will be the state of exposure science 10 years from now? In considering this question, it is hard to imagine sensor technologies not being an important theme. We believe that sensor technologies do, and will continue to, define the cutting edge of exposure science, now and in the future.

The meteoric rise in accessible technology has improved lives in countless ways, from smart thermostats that improve energy efficiency and comfort to automobiles equipped with countless sensors to support autonomous driving and everything in between. As exposure scientists and environmental epidemiologists, we are driven by data to inform health protective decisions, and these technologies can now be harvested to provide previously unattainable information describing our environment.

There are four intertwined characteristics of sensors that together ensure that this technology is likely to play a prominent role at the cutting edge of exposure science. This special issue, entitled 'Sensing for Exposure Science' in the Journal of Exposure Science and Environmental Epidemiology highlights a number of important advancements in sensors.

First, sensors provide measurement capability. While measurements are the life blood of exposure science, collecting measurements of exposure is often resource intensive-equipment is scarce and expensive, measurements are sometimes viewed as too invasive, or it is simply impractical to measure exposure because of the complexity of human behavior [1]. But new sensor technologies have short-circuited many of these challenges, and scientists now have access to a vast array of sensor technologies that

Richard E. Peltier

rpeltier@schoolph.umass.edu

University of Massachusetts, Amherst, MA, USA

2 U.S. EPA/ORD's Center for Public Health \& Environmental Assessment, Research Triangle Park, NC, USA provide space, time, and behavior resolved measurements that were previously unattainable.

Second, sensor technology provides the means for personal measurements, i.e., capturing the time and space continuum of the individual. The human requirements for personal measurements preclude use of most traditional measurement instrumentation designed for outdoor and indoor monitoring. Personal measurements need to meet human requirements related to noise, weight, wear ability, appearance, and battery life so as not to become a barrier to, or bias human subject recruitment or alter behavior, i.e., the Hawthorne effect. Sensor technologies "thread the needle" of requirements for personal monitoring thereby enabling this uniquely valuable measure of individual exposure.

Third, sensor technology and the measurements it provides hold the potential for vast consumer availability and use. This potential exists because sensors are generally relatively inexpensive and the information they provide has broad consumer appeal. Imagine the air pollution equivalent of Fitbit. Manufacturers have developed many sensing devices that are tailored to consumer purchase and use including internet networking and social considerations allowing comparison and competition among family and friends.

Fourth, sensor technology will enable linkages to transformations occuring within medicine. These transformations include personalized medicine, electronic records, and growing recognition of the importance of environment in influencing disease. There is growing awareness within medicine of the importance of environmental factors that cause or exacerbate disease. Asthma [2], chronic obstructive pulmonary disease [3], and cardiovascular disease [4] being prime examples. We are not far from the day when medical evaluation and treatment of these diseases will include environmental sensors to track with symptoms and/ or environmental interventions such as HEPA air cleaners.

Exposure and related sciences will need to pave the way to this future. The implications of using novel sensors to assess our world are still not fully understood and open questions remain unanswered. How do we evaluate the 
precision, accuracy, reliability and fit for purpose? What does the influx of extreme granularity of data tell us as exposure or health scientists? Are there unanticipated ethical concerns with such data? These questions are cautionary, but also demonstrate enticing possibilities, as sensors continue their pervasive growth in use and application.

At the same time, sensors bring forth the potential to democratize exposure science. Sensor technologies are enabling and empowering the general public and citizen scientists [5]. They hold the potential to reveal environmental injustices and provide supporting data to hold responsible parties accountable [6]. The technology is enabling in its affordability, ease of use, and social engineering. It is empowering in providing the user with data that can inform exposure-mitigating behaviors or as a basis of advocating for community environmental health interests among relevant state or local government agencies [7].

In this issue, we see the breadth and depth of sensor use and methodological development that spans across the globe. It provides a robust snapshot of the diverse and varied approaches in which sensors can provide actionable data leading to health protective changes. In this special issue, these topics are delineated into four broad categories of work: associations between sensor measurements with health endpoints, characterizing microenvironments of individuals, and two areas of assessment in locations where there is limited data-one that evaluated traffic-related pollutants, and another assessing clean cook stove efficacy and policy.

These studies provide a far more complex and nuanced understanding of exposure. Diverse and flexible applications of sensors let us investigate disease causality in ways never possible until now. For example, sensors can be worn by individuals as a person goes about their day or can be more easily shipped to locations where no measurement infrastructure exists. As a result, these are instruments of high utility that can be adapted and improvised into new and exciting study designs.

At the same time, the growing use of sensors introduces new questions to exposure-related research that provide an important and often overlooked connection to environmental epidemiology. For example, how do we manage measurements of a pollutant collected at $1 \mathrm{~s}$ intervals over the longer time-averaged equivalent more commonly used by epidemiology? Is this level of detail helpful to understanding disease and causality, or is it unnecessary? How do sensor measurements, which are usually available with less precision and accuracy, relate to more robust measurements from a reference monitor, even though that reference monitor might be located far from the environment under study?

The studies published here are very much exemplify sensor technologies at the cutting edge of exposure science. These studies demonstrate both remaining technical limitations, as well as open up the possibility to answer new questions in creative ways. The results in this special issue are illustrative of the success of sensors technologies meaningfully contribution to exposure science.

As we look to the future of exposure science and the challenges of a habitable planet, we anticipate that sensor technologies will be at the cutting-edge providing a critical capability in a feedback loop to protect human health and the environment.

\section{Compliance with ethical standards}

Conflict of interest The authors declare that they have no conflict of interest.

Publisher's note Springer Nature remains neutral with regard to jurisdictional claims in published maps and institutional affiliations.

\section{References}

1. National Research Council. Exposure science in the 21st century: a vision and a strategy. Washington DC, USA; National Academies Press; 2012.

2. Zheng XY, Ding H, Jiang LN, Chen SW, Zheng JP, Qiu M, et al. Association between air pollutants and asthma emergency room visits and hospital admissions in time series studies: a systematic review and meta-analysis. PLoS ONE. 2015;10:e0138146. https:// doi.org/10.1371/journal.pone.0138146.

3. Rosenberg SR, Kalhan R, Mannino DM. Epidemiology of chronic obstructive pulmonary disease: prevalence, morbidity, mortality, and risk factors. Semin Respir Crit Care Med. 2015;36:457-69. https://doi.org/10.1055/s-0035-1555607.

4. Bhatnagar A. Environmental determinants of cardiovascular disease. Circulation Res. 2017;121.2:162-80.

5. Jerrett M, Donaire-Gonzalez D, Popoola O, Jones R, Cohen RC, Almanza E, Nazelle A, et al. Validating novel air pollution sensors to improve exposure estimates for epidemiological analyses and citizen science. Environ Res. 2017;158:286-94.

6. Kaufman A, Williams R, Barzyk T, Greenberg M, O'Shea M, Sheridan $\mathrm{P}$, et al. A citizen science and government collaboration: developing tools to facilitate community air monitoring. Environ Justice. 2017;10:51-61.

7. Barzyk TM, Huang H, Williams R, Kaufman A, Essoka J. Advice and frequently asked questions (FAQs) for citizenscience environmental health assessments. Int J Environ Res Public Health. 2018;15:960. https://doi.org/10.3390/ ijerph15050960. 